\title{
Sociología del arte de Marcel Duchamp
}

\author{
Tomás Peters Núñez*
}

\begin{abstract}
Resumen
El presente artículo realiza una breve discusión sobre cómo la sociología y el arte se aportan mutuamente para la comprensión e interpretación de las manifestaciones de la modernidad. Con el objetivo de aportar elementos a esa discusión, se realizó una revisión bibliográfica sobre los análisis desarrollados por la sociología en torno a la obra del artista francés Marcel Duchamp. En una primera parte del artículo, se entregan elementos generales de lo dicho por la sociología sobre el artista dadaísta. En una segunda parte, se presentan los análisis que realizaron los sociólogos Zygmunt Bauman, Pierre Bourdieu y Niklas Luhmann sobre la obra del artista y de cómo ella sirve para comprender la modernidad.

Palabras clave: Duchamp, sociología, arte, modernidad, conocimiento
\end{abstract}

\begin{abstract}
This article presents a brief discussion about how the sociology and the art contribute to the understanding and interpretation of the manifestations of modernity. In order to provide elements to this discussion, this article reviews the literature on the analysis developed by sociology about the work of the French artist Marcel Duchamp. In the first part of this paper, we present general elements that sociology has thought about the Dada artist. In the second part, we present the analysis conducted by sociologists Zygmunt Bauman, Pierre Bourdieu and Niklas Luhmann on the artist's work and how it helps to understand the modernity.
\end{abstract}

Key words: Duchamp, sociology, art, modernity, knowledge

* Chileno. Sociólogo e investigador del Centro de Investigaciones Socioculturales de la Universidad Alberto Hurtado. tpeters@uahurtado.cl 


\section{Introducción}

En la década de los ochenta, se planteaba que entre la sociología y el arte no existía una amistad recíproca (Bourdieu, 2002). Por el contrario, entre ellas existía una tensión y cuestionamiento permanente, debido, principalmente, a la idea que cada una de ellas tenía de la otra: la sociología desvelaba la creencia del don artístico y el arte, por su parte, criticaba el reduccionismo analítico realizado por la sociología a la creación artística. Con el pasar de los años, y especialmente durante la década de los noventa, esta condición varió sustancialmente: las mutuas instancias de diálogo y reflexión dieron paso a un nuevo trato (Heinich, 2001 y 2002).

Si bien la reflexión filosófica entre arte y sociedad posee una tradición importante (Adorno y Horkheimer, 2003; Benjamin, 2003; Francastel, 1975; Hauser, 1983; Duvignaud, 1988; Rancière, 2009), recién en los últimos veinte años los estudios sociológicos dedicados al arte han experimentado una creciente e importante atención (Wolff, 1997; Harrington, 2004). Con ello, las actuales reflexiones sociológicas y estéticas ya se han acostumbrado a compartir ciertas observaciones y, en más de alguna ocasión, a discutir conceptos y teorías que parecían antagónicas y excluyentes entre cada disciplina (De la Fuente, 2000; Pollock, 2007). Entre ambas, se ha generado un diálogo fecundo para comprender e interpretar las problemáticas de la modernidad (Rojas, 2003).

En Chile -y en general en América Latina- se conoce poco el rendimiento analítico que provee la sociología del arte para comprender e interpretar las manifestaciones de la modernidad (García Canclini, 1979 y 2010; Facuse, 2010). Esto ha implicado, entre otras cosas, devaluar el análisis crítico del arte como una herramienta productiva para la reflexión sociológica contemporánea. Es más, ha impedido desarrollar una sociología capaz de hacerse cargo, crítica y responsablemente, de la dimensión estética -visual, sonora, poética- de la sociedad latinoamericana. En consideración a aquello, resulta central entender la esfera del arte 
como un espacio cultural que produce una reflexión propia acerca de lo social -un conocimiento "otro"-, que puede enriquecer la reflexión sociológica.

El presente texto intenta hacer frente a esta situación. Con el objetivo de demostrar que el arte no es un espacio independiente de la sociedad, sino que, por el contrario, es un conocimiento otro que permite comprender a la modernidad y sus múltiples dimensiones, este artículo desarrolla un ejercicio concreto por observar cómo algunos autores contemporáneos de la sociología se han apoyado en el arte para interpretar a la modernidad. Basándose en las propuestas teóricas de Zygmunt Bauman, Pierre Bourdieu y Niklas Luhmann, el artículo realiza una breve sistematización de las observaciones/interpretaciones realizadas por ellos en torno a la obra del artista francés Marcel Duchamp.

En la primera parte del texto se presenta una descripción resumida de las obras - $y$ acciones- realizadas por Marcel Duchamp en la primera mitad del siglo XX, para, con ello, esbozar un breve análisis introductorio que algunos sociólogos del arte menos reconocidos dedicaron a la figura del artista francés. En una segunda parte, se presentan las interpretaciones específicas de los tres sociólogos antes anotados, con respecto a la importancia de Duchamp para comprender ciertas transformaciones que ha sufrido la modernidad en el siglo XX. En una tercera parte, se presenta una síntesis de estas observaciones y se plantean algunos nudos críticos surgidos en esta discusión.

\section{Observaciones sociológicas generales de la obra de Marcel Duchamp}

En 1913 Marcel Duchamp (francés, 1887-1968) mostraba, en la Armony Show de Nueva York, su ejercicio pictórico Desnudo bajando una escalera $N^{\circ}$ 2. La obra, de inspiración cubofuturista, causó cierto cuestionamiento al interior del "mundo del arte", debido a su provocativo rechazo, entre otras cosas, del realismo figurativo convencional (Lyotard, 1977). En aquel esfuerzo, Duchamp 
lograba poner al tapete la problemática del movimiento en el arte de inicios del siglo XX y, sobre todo, la noción de mecanismo como elemento posible de representación (Ramírez, 2000). De aquella matriz, vendrían obras como El gran vidrio y El molinillo de café, las que desembocarían en nuevas estructuras reflexivas sobre la ciencia, la sexualidad y la cuarta dimensión, entre otras cosas. En ese mismo año (1913), Duchamp comenzaría con uno de los proyectos artísticos que marcaría profundamente la trayectoria del arte moderno: Rueda de bicicleta sobre un taburete. Tal propuesta sería, sin lugar a dudas, la génesis del cambio paradigmático del arte actual, ya que mostraría que cualquier cosa podría, según ciertas reglas internas del campo artístico, lograr la posibilidad de ser una obra de arte (Danto, 2006). Colocando una rueda delantera de bicicleta dispuesta bocabajo sobre un taburete de cuatro patas, la obra presentaría un cuestionamiento claro a las expresiones tradicionales de la tri y bidimensionalidad $\mathrm{y}$, sobre todo, a la expresión clásica de lo bello (Ramírez, 2000). Con ello, surgiría la noción de readymade, es decir, que cualquier producto industrial podía convertirse en una obra de arte a merced de un artista, pero que, además, fuera imposible de apreciar y describir en términos estéticos bajo la lógica bello/no bello.

Posteriormente, serían dos obras las que, siguiendo la lógica del readymade, absorberían la atención no sólo del mundo artístico, sino que de todo el espacio de reflexión moderno: $L H O O Q$ (1919) y Fuente (1917). En el primer caso, Duchamp dibujaría, sobre una reproducción de la Mona Lisa de Leonardo, un simple y disonante bigote en su rostro. Además escribiría, en un juego de palabras del francés, un título poco convencional para aquellos años. ${ }^{1}$ En el segundo caso, Duchamp enviaría a una exposición de arte modernista un urinario común y corriente, cuya firma -R.Mutt- e idea de cuestionamiento, tendría gran repercusión en todos los frentes dedicados a la institucionalidad artística moderna (Danto, 2006; Shiner, 2004). En ambos casos, la idea estaba

1 L.H.O.O.Q. (1919): “Ella tiene el culo caliente”. 
clara: es posible, en base a las lógicas del arte moderno, jugar con sus normas, condiciones e interpretaciones. De esta forma, y en casi una década de historia, la trayectoria de lo que se entendía por Arte cambiaría considerablemente.

Estas obras -que sirven como excusa general para explicar el contexto de este artículo- han dado paso a múltiples interpretaciones analíticas. Una obvia y general es observable, por ejemplo, en el formalista de Gombrich (2007: 601): "El artista francés Marcel Duchamp adquirió fama y notoriedad en base a coger cualquier objeto (al que él llamaba ready-made [ya-hecho]) y firmarlo". Sin embargo, es posible reconocer, en el trabajo del artista francés, una serie de observaciones sociológicas que resultan interesantes y dignas de considerar como complementarias a cualquier ejercicio estético. Veamos, brevemente, algunas reflexiones desarrolladas en las últimas décadas.

Howard Becker (2008) ha planteado, en base a la teoría institucional -que apunta a resolver los problemas que presentan los trabajos que atentan contra el sentido común y las sensibilidades más refinadas, al no manifestar rastro alguno del artista-, que el trabajo artístico, como toda labor social, exige de una acción colectiva mediada por normas y patrones establecidos. Es decir, que las múltiples interacciones que dieron paso a la producción, mediación y recepción de una obra de arte, constituyen lo que él denomina como "mundos del arte". En sus palabras, "los mundos del arte consisten en todas las personas cuya actividad es necesaria para la producción de los trabajos característicos que ese mundo... define como arte. Los miembros de los mundos del arte coordinan actividades por las cuales se produce el trabajo haciendo referencia a un cuerpo de convenciones que se concretan en una práctica común y objetivos de uso frecuente" (Becker, 2008: 54). Con ello, Becker propone que no es posible comprender una obra sin considerar el entramado de relaciones sociales históricas que en ella existió. De ahí que, para él, la obra de Duchamp tenga un especial interés, ya que, en su conjunto, manifiesta un claro cuestionamiento a la producción 
artística como algo nacido gracias a la inspiración sublime, es decir, único y sólo posible por la genialidad del artista. En desmedro de aquella posición tradicional, Becker explica que "Marcel Duchamp violó la ideología al insistir en que había creado una obra de arte legítima cuando firmó una pala de nieve de producción comercial o una reproducción de la Mona Lisa en la que había dibujado un bigote, clasificando así a Leonardo como personal de apoyo junto con el diseñador y el fabricante de la pala de nieve" (Becker, 2008: 37). En este sentido, el trabajo de otros podía contribuir, mediante una intervención o recomposición de escena, a la creación de un objeto considerado artístico. Y éste mismo podía contribuir, a la vez, en nuevas creaciones, posibilitando diversos despliegues reflexivocomunicativos en el arte moderno.

Sumado a lo anterior, Becker planteaba que gran parte de la legitimidad de la obra de Duchamp estaba lograda gracias a la firma que cada objeto poseía de él (argumento similar al propuesto por Gombrich). Es decir, que, colocando en una balanza la calidad y la condición artística del trabajo con respecto a las organizaciones en las que el arte se produce, distribuye, aprecia y analiza mediante el reconocimiento de un autor, sería la firma la más importante al momento de juzgar lo que pertenece o no al "mundo del arte". Sería, finalmente, la relación existente entre la obra, el artista y las diversas instancias sociales institucionales la que otorgaría, en el contexto moderno, lo digno de llamarse Arte.

Esta primera aproximación de la sociología del arte entregaría ciertos lineamientos generales de la obra de Duchamp, pero dejaría de lado las implicancias que este ejercicio significaría para el mundo moderno general y, específicamente, para el mundo del arte que Becker proponía.

Vera Zolberg (2002), por su parte, plantearía, en su Sociología de las artes, que el artista francés "habiendo iniciado su carrera como un pintor que se entretenía con diversos estilos avanzados... no limitó su travesura al viejo establishment, sino que también indignó al 
mundo del arte moderno" (Zolberg, 2002: 40). Es decir que no sólo las propuestas artísticas de Duchamp desagradarían al público clásico del arte (críticos, marchantes, etc.), sino que también a los que junto a él caminaban por la senda de la ruptura con el arte anterior (especialmente al del siglo XIX): "el respetable mundo artístico vanguardista, la propia fuente de su promoción, también se sentía horrorizada-o, al menos, desagradablemente afectada- por su comportamiento" (Zolberg, 2002: 40). Esta línea analítica es evidente en el caso de Fuente de Duchamp. En 1917 Duchamp pertenecía al consejo de la "Sociedad de Artistas Independientes", el cual había organizado una exposición anual y donde "cualquier artista" podía participar. La exposición, que no entregaría premios ni tendría jurados, motivó a Duchamp a poner a prueba a sus colegasartistas, firmando un urinario bajo el seudónimo ya mencionado de R. Mutt. Los resultados fueron, para Duchamp, clarificadores: el consejo rehusó exponer a Fuente, debido a que se trataba de una pieza obscena -que no era arte- y que "pondría en ridículo a la Sociedad de Artistas Independientes" (Shiner, 2004: 392).

Esto se vería reflejado en el cuestionamiento de lo que era, hasta ese momento, considerado un objeto de arte. En este sentido, el resultado de los incesantes ataques a los diversos significados del arte que realizó Duchamp abrió la puerta, al menos hasta la actualidad, a la posibilidad de que prácticamente cualquier cosa pueda ser presentada con la pretensión de ser una obra de arte, dejando de lado, por tanto, toda consideración preconcebida de artista. Más aún, que cualquier objeto sea posible de ser institucionalizado como obra, plantea el profundo cuestionamiento del rol del artista como creador de nuevos espacios de representación crítica. Si bien el trabajo de Duchamp lograba desestabilizar la noción de obra, también lo hacía, directa o indirectamente, a la de artista mismo. Esto causó, ciertamente, entre sus propios contemporáneos, el cuestionamiento de todo proceso lógico de creación: el pincel y la mancha libre del autor, daban paso a nuevos patrones de producción y reconocimiento artístico. 
Este proceso, según la observación de Aaron Panofsky (2003), causaría una ruptura epistemológica de alto impacto. En primer lugar, la propuesta de Duchamp realizaría una profunda crítica institucional (artístico-museal) que propiciaría una transformación de la agenda programática de los patrones de conocimiento y comprensión. Además, y como segundo lugar, la propuesta del artista francés reconfiguraría los patrones de observación metodológica de lo considerado cierto o verdadero (desconcertando las estructuras científicas de conocimiento). Y, en tercer lugar, propondría, con sus trabajos, una nueva posibilidad de reflexión en la estructura intelectual moderna, logrando destapar las rígidas arquitecturas de comprensión del todo.

Frente a este panorama, la obra de Duchamp nos demuestra que, más allá de sus propuestas estéticas y artísticas, causó una serie de resquebrajamientos en las estructuras convencionales de comprensión de lo cierto y legítimo que, hasta ese momento -inicios del siglo XX- venía ofreciendo la sociedad moderna. Las obras, al ser consideradas desde una perspectiva sociológica, permiten ser vistas como gestos informativos -conocimientos- que ofrecen antecedentes comprensivos de las nuevas formas de organización y re-formulación que la sociedad moderna despliega para su habitar. En este sentido, la sociología del arte ha logrado comprender e interpretar ciertos procesos de transformación de la sociedad moderna a partir de los procesos de producción, mediación y recepción artística (Furió, 2000).

Los trabajos sociológicos realizados por Zygmunt Bauman, Pierre Bourdieu y Niklas Luhmann, sirven para respaldar el punto anterior. Sus planteamientos, que sirven como puentes analíticos entre arte y sociedad, nos permiten analizar cómo sus modelos teóricos se ven beneficiados, empíricamente, con las obras de Marcel Duchamp. Con ello, es posible comprender a la modernidad a partir de una manifestación artístico-cultural (obra) que propone y explica conflictos, representaciones y disonantes formas de conocimiento en la sociedad. 


\section{Bauman, Bourdieu y Luhmann: sociología del arte de Marcel Duchamp}

La sociología del arte se configura como perspectiva analítica a partir de una raíz teórica general. Es decir, ella no nace como disciplina independiente de las grandes corrientes sociológicas y, menos aún, prescinde de ellas. Por esta razón, y para iniciar una reflexión general sobre la relación entre sociología y arte, es relevante profundizar el análisis arriba iniciado con tres modelos sociológicos contemporáneos: la teoría de la modernidad líquida de Bauman, la teoría de los campos de Bourdieu y la teoría de sistemas de Luhmann. Los tres han abordado la obra de Duchamp -con diferentes énfasis- a partir de sus propios modelos teóricos y han utilizado su obra como material empírico para comprender e interpretar a la modernidad.

\subsection{Bauman: Los readymade de Duchamp como arte, ¿líquido?}

Para Zygmunt Bauman (2003), la modernidad, como proyecto, estaría en cuestionamiento o, simplemente, puesta en duda como posibilidad alcanzable. Según sus postulados, las transformaciones sociales acaecidas en el siglo XX habrían marcado un panorama general de constante fluidez y de profunda liberalización (flexibilización) de las relaciones sociales. En sus propias palabras, la “disolución de lo sólido, el rasgo permanente de la modernidad, ha adquirido por lo tanto un nuevo significado, y sobre todo ha sido redirigida hacia un nuevo blanco: uno de los efectos más importantes de ese cambio de dirección ha sido la disolución de las fuerzas que podrían mantener el tema del orden y del sistema dentro de la agenda política" (Bauman, 2003: 5). En este sentido, las consideraciones generales de esta nueva etapa significarían el traslado de marcos de referencia sólidos (estructurados institucional e históricamente) a referentes cada vez más dispersos y abiertos -fluidamente- a nuevas propuestas, situando así una sociedad de la contingencia. Estos cuestionamientos darían paso a considerar la vida actual, entre otras 
terminologías, como posmoderna (Lyotard, 1998; Huyssen, 2002; Foster, 2001).

Sin embargo, el actual contexto sería, para Bauman, un escenario posterior al de Duchamp. Para el sociólogo polaco, la obra del artista francés tendría un cariz distinto a toda la escena posmoderna. Ella sería, según sus postulados, la mayor manifestación moderna del arte moderno: "Retrospectivamente, el acto escandaloso de Marcel Duchamp, que en su momento se consideró como un desafío descarado a virtualmente todo lo que simbolizaba la estética occidental, parece asombrosamente moderno más que posmoderno; lo que Duchamp hizo fue proponer una nueva definición del arte (algo elegido por el artista), una nueva teoría de la obra de arte (separar un objeto de su contexto habitual y verlo desde un punto de vista insólito: hacer de hecho lo que los románticos habian efectuado un siglo antes al convertir lo familiar en extraordinario) y un nuevo método de trabajo artístico (crear una nueva idea para un objeto)" (Bauman, 2005: 187). En este sentido, se puede decir, con claridad, que el gesto de Duchamp no fue en absoluto iconoclasta, sino que por el contrario: erigió el proyecto del arte moderno. En otras palabras, ¿qué es más moderno que el cuestionamiento de las estructuras dominantes totales? ¿No es acaso parte del proyecto moderno el cuestionamiento de las definiciones, teorías y métodos que se establecen como preponderantes del juicio estético? En este panorama, Bauman anota que Duchamp logró oponerse e impugnar lo que, hasta ese momento, resultaban ser las reglas del juego. Y esas reglas del juego eran, hasta ese momento, incuestionables.

El arte posmoderno se caracterizaría, según Bauman, por la ausencia de un estilo (Bauman, 2007). Su carácter es, claramente, ecléctico: alejado de todo estilo dominante, pureza de género, escuelas, etc. Por ello, toda posibilidad de cuestionamiento no es posible, ya que resulta imposible toda innovación o ruptura con respecto al todo (serían negaciones nimias o irrelevantes entre todos los estilos convivientes). En sus palabras, en el arte posmoderno "ya no hay ningún desarrollo en arte; tal vez, sólo un cambio sin 
dirección, una sucesión de modas, en las que ninguna forma sostiene de manera creíble su superioridad sobre las predecesoras, las cuales, por la misma razón, se convierten en sus contemporáneas" (Bauman, 2005: 186). En este escenario, el trabajo de Duchamp lo que hizo fue enaltecer el proyecto moderno: más bien, hacerlo visible. Recordarnos que el arte, por fin, puede ser parte de un proyecto de libertad, donde exista una oportunidad de reinventar el mundo. Por ello, el ready-made estaría siendo la propuesta artística que gatilló el quiebre con la estructura dominante y dio paso, además, a la amplitud de posibilidades creativas y reflexivas de las cuales hoy somos testigos. En otras palabras, Duchamp fue la génesis moderna de la actualidad posmoderna. Pero, sin lugar a dudas, su obra es y será siendo un ejemplo puro de lo que por décadas se ha comprendido como arte moderno.

\subsection{Bourdieu: ¿Quién creó a Duchamp?}

Pierre Bourdieu (1995), por su parte, interpreta la sociedad moderna por la génesis de estructuras sociales autónomas denominadas campos. Existiría, para él, una amplia diversidad de campos y subcampos que funcionan con una lógica específica pero que, a su vez, comparten un conjunto de leyes generales, válidas para todos. Cada campo específico se define a partir del capital que en él está en juego. El capital puede definirse como un conjunto de bienes acumulados que se producen, se distribuyen y se consumen. El capital simbólico, por ejemplo, es la acumulación de todas las especies de capitales posibles, que generan crédito y autoridad en los agentes que la poseen. La fuerza de dicho capital reside en la significación que toman los atributos de los agentes por medio de un trabajo permanente de legitimación que se funda en la transformación de las diferencias de hecho.

El campo artístico, y específicamente el caso de las artes visuales, estaría constituido, para Bourdieu, por las relaciones entre artistas, galeristas, críticos, teóricos, curadores, historiadores y su público, en el entendido de que lo que articula el campo no son las relaciones subjetivas entre los individuos que ostentan ta- 
les títulos, sino entre los puestos estratificados (jerarquizados) en los que están permanentemente intentando consumir, inscribirse y, sobre todo, legitimarse (Bourdieu, 2003a y 2000). Por ello, las prácticas y representaciones de los artistas sólo pueden explicarse por referencia al campo de poder. Para Bourdieu, el campo de poder "es el espacio de las relaciones de fuerza entre agentes e instituciones que tienen en común el poseer capital necesario para ocupar posiciones dominantes en los diferentes campos (económico y cultural en especial). Es la sede de luchas entre ostentadores de poderes diferentes..." (Bourdieu, 1995: 321-322).

Desplegando así una tesis central en este campo, el sujeto de la obra de arte no es el artista, sino todo el conjunto del campo de la producción artística (Bourdieu, 1997). Un artista existe como tal a partir de su capacidad para lograr que se le reconozca como ocupante de una posición en el campo, en relación con la cual tendrán que situarse los demás. Es decir, se trata tanto de hacerse visible como legible a partir de los términos de las grandes oposiciones que sirven para concebir la lucha que articula al campo. Tal como lo plantea Bourdieu: "No hay campo donde el enfrentamiento entre las posiciones y las disposiciones sea más constante y más incierto que el campo literario y artístico, el campo de producción cultural constituye el terreno por excelencia de las luchas para la redefinición del puesto" (Bourdieu, 1997: 318). Sin embargo, para lograr las posiciones jerárquicas en el campo artístico se requiere de un habitus que le permita desplegar estrategias concretas para ese fin. En este sentido, cada puesto alcanzado dependerá de las formas de sentir, actuar y pensar que son heredadas socialmente y que permiten adueñarse de las posiciones hechas o hacer nuevas posiciones (Bourdieu, 2000).

En este escenario, Marcel Duchamp juega un papel fundamental. Él sería, para Bourdieu, un ejemplo concreto de un agente estratégico que utiliza las reglas del juego (del arte) para dinamizar el propio campo artístico. Cumpliría con el paradigma de artista que se las sabe todas por excelencia: el artista que rompe 
continuamente con las convenciones, incluso con las de la vanguardia. En las palabras del sociólogo francés, "Conociéndose el juego al dedillo, (Duchamp) produce objetos cuya producción como obras de arte supone la producción del productor como artista: inventa el ready-made, ese objeto manufacturado promocionado a la dignidad de objeto de arte mediante un empujón simbólico del artista" (Bourdieu, 1997: 367). Y ese gesto, tan simbólico y simple -como evidenciar aspectos ocultos de los objetos al aislarlos del contexto familiar de donde proceden su significación y sus funciones habituales-, resultaría ser la estrategia más efectiva observada en el arte moderno. Es más, Duchamp jugaría a tal punto con este actuar, que incluso podía poner en cuestión, permanentemente, sus intenciones discursivas, logrando, con ello, jugar aún más con el juego de las posiciones jerárquicas de poder: "Pero sobre todo, como buen jugador de ajedrez que, dueño de la necesidad inmanente del juego, puede inscribir en cada jugada la anticipación de las jugadas sucesivas que va a poner en marcha, Duchamp prevé las interpretaciones para desmentirlas o desbaratarlas" (Bourdieu, 1997: 338). Pero esto se logra gracias al conocimiento y reconocimiento de las leyes inmanentes del campo. Por ello, se considera a Duchamp como un "Virtuoso en el arte de jugar con todas las posibilidades que ofrece el juego", ya que él "simula que retorna al mero sentido común para denunciar las interpretaciones rebuscadas que los críticos más escrupulosos han dado de sus obras; o bien deja que planee la duda, mediante la ironía o el humor, sobre el sentido de una obra deliberadamente polisémica" (Bourdieu, 1997: 338).

Para Bourdieu el gesto artístico de Duchamp podría catalogarse, en síntesis, como el acto artístico supremo de la modernidad, ya que cambiaría radicalmente la configuración/estructura interna e histórica del campo artístico moderno, en base a la conformación de nuevas categorías de desciframiento del arte, como también de la instauración de lo que es o no legítimo de considerar como obra de arte en el contexto moderno. La obra de arte, en definitiva, sólo existe como objeto simbólico provisto de valor, si es conocida y está reconocida; es decir, si está socialmente instituida como 
obra de arte por unos espectadores dotados de la disposición y por la competencia artística necesaria para conocerla y reconocerla como tal (Bourdieu, 2003b). Por lo tanto, para desarrollar una teoría adecuada del campo, es necesario considerar la importancia de los agentes sociales, los cuales están dotados de un conjunto de disposiciones que implican propensión y capacidad de entrar en el juego y poder jugar en él para así cambiarlo desde adentro, tal como lo hiciera Duchamp durante el siglo XX.

\subsection{Luhmann: el arte como mundo en Duchamp}

Para el sociólogo alemán Niklas Luhmann (2005), el avance (evolución) de la sociedad moderna se caracterizaría por la diferenciación de funciones en base a criterios específicos de organización y descripción: el derecho, la economía, la política, el arte, la ciencia, etc., se podrían describir como sistemas funcionalmente diferenciados (Luhmann, 1982, 2007). Este proceso implicaría el aumento progresivo de la complejización social, lo que contribuiría a la conformación de estructuras de funcionamientos autónomos y auto-organizados.

Para comprender la observación de Luhmann sobre Duchamp es importante responder las siguientes preguntas: ¿Cómo comprender la emergencia de un sistema artístico moderno? O mejor dicho: ¿Cómo se comprende teóricamente el arte desde la sociología del arte de Niklas Luhmann? La respuesta debe comprenderse por un proceso evolutivo complejo (Luhmann, 2005), del cual no sólo el arte ha sido parte de él, sino que todos los demás sistemas sociales (sin excepción).

Para Luhmann, "El arte moderno debió encontrar la ocasión de adaptarse a la sociedad moderna y por eso tuvo que reformular su referencia al mundo" (Luhmann, 1993: 13). Y cuando el arte reformula su referencia al mundo, el arte se entiende como un observador que exige el ser observado. Pues bien, ¿qué exige el ser observado? Luhmann respondería: se observa la producción de distinciones (la observación de la observación). 
Para comprender lo anterior, el arte moderno exige revisar la distinción entre observación de primer orden y de segundo orden. Ambas distinciones son, para Luhmann, parte fundamental del arte moderno y, especialmente, para comprender la obra de Marcel Duchamp. Para el sociólogo alemán, una observación de primer orden describe un "objeto de arte": la escultura es de madera, la perfección del pulido, la circunferencia perfecta de la pintura, etc. Un observador del renacimiento italiano observaría, por ejemplo, un bello paisaje cercano a su pueblo, el cual le hace sentido a su percepción. En cambio, el observar un urinario en un espacio museal o una rueda de bicicleta colocada sobre un retrete, exige una nueva observación, que ofrezca nuevas posibilidades de comprensión: una observación de segundo orden. Ella, para Luhmann, describe "la distinción por medio de la cual lo distinto resalta como diferencia de lo otro" (Luhmann, 1993: 13).

En otras palabras, la observación de segundo orden observa las distinciones que propone la obra y de qué manera están orientadas las unas a las otras. Este observador de segundo orden logra, por tanto, observar la distinción de lo que funciona/ no funciona, lo que encaja/no encaja en la obra instalada por Duchamp, pero nunca excluye una obra de arte por esta distinción. Por el contrario, logra establecer la distinción necesaria sobre qué comunicación específica desea realizar la obra misma. En palabras de Luhmann: "La obra de arte puede ser señalada como artística y re conocida -en los museos, en las galerías, en los estudios, en las salas de concierto, en el teatro- a través de los anuncios de las editoriales o a través de los nombres célebres de los artistas. Sin embargo, también esto es un problema a tomarse en serio principalmente desde que artistas como Marcel Duchamp o John Cage se ha encaprichado en excluir para esta pregunta toda distinción sensorial notoria (con excepción de su nombre), y así confrontar al observador con la interrogación acerca de cómo hace realmente para reconocer una obra de arte como arte. Y como única respuesta queda entonces la siguiente: mediante una observación de la observación, mediante la observación sobre la disposición del artista quien está orientado a llamar la atención 
sobre sí mismo rechazando todas las demás distinciones como irrelevantes" (Luhmann, 2005: 124).

Para Luhmann, entonces, esta distinción permite observar la conformación de una obra de arte moderna, ya que nos ofrece la posibilidad histórica de distinguir los esquemas que ella misma intenta comunicar. De ahí que la obra de Duchamp sea útil para los propósitos de Luhmann, ya que su trabajo exige desarrollar una observación novedosa: no la necesaria para reconocer un objeto de porcelana ovalado que sirve para algo. La observación que exige la obra de Duchamp es, por el contrario, la que permita comprender su lado $b$. Es decir, identificar la comunicación que la obra plantea ya no sólo en sus condicionantes materiales, sino que también en la posibilidad que ella genera para una mayor reflexión futura. De esta forma, y por medio de textos o relatos que inviten a ampliar los márgenes de posibilidad comunicativa de cada expresión visual, sonora o literaria, el sistema artístico podrá complejizar su operar y ofrecer, por tanto, nuevas obras artísticas cada vez más radicalizadas en su experimentación representacional.

En resumidas cuentas, para comprender la obra de arte, el espectador/lector/público debe, luego de reconocer las características del mundo que propone la obra, distinguir el esquematismo o el juego de distinciones desde el cual esta particular obra está reconstruyendo/describiendo/observando el mundo. El espectador debe así observar el o los puntos de vista que estructuran la obra y dan sentido a las formas que en ella se proponen, dejando de lado la observación obvia.

Pero este orden de posibilidad que logra la obra de arte va configurando, en su inmensa complejidad, un sistema autorreferente que logra estabilizar, comunicativamente, la semántica del arte moderno. Por ello, para comprender la sociedad moderna, debemos tener presente, en todo momento, que el aumento de complejidad logrado por la obra de Duchamp, permitió nuevos despliegues auto-reflexivos y críticos en el arte moderno, logran- 
do con ello nuevos espacios de autonomía sistémica (Luhmann, 2005).

\section{Observaciones finales sobre un tablero de ajedrez}

Los tres modelos sociológicos presentados en este artículo demuestran, en términos generales, que cada uno de ellos pone énfasis distintos al momento de analizar la obra de Marcel Duchamp. En este sentido, la obra por sí sola ofrece una multiplicidad de opciones analíticas que han permitido a la sociología desplegar variadas interpretaciones sobre la relación entre arte y sociedad moderna. Considerando las disonancias y elementos en común, los tres modelos teóricos lograron desplegar, a partir de sus propias arquitecturas teóricas, esquemas de sentido novedosos para comprender la complejidad de la modernidad.

La obra de Marcel Duchamp, por tanto, ha servido como un importante insumo analítico a la sociología. Sus propuestas y acciones, tan cuestionadas y ensalzadas a la vez, han exigido la creación de propuestas analíticas -observaciones- complementarias a la estética. Si bien la obra misma dispone de cualidades estéticas indudables, lo interesante de anotar aquí es que ella ha traspasado las fronteras disciplinarias del arte y ha permitido su uso para interpretar la modernidad. Esto es, sin dudas, la fortaleza de Duchamp. Por ello, la revisión que los autores de la sociología del arte realizaron sobre Duchamp, contribuye a la ampliación de la discusión posible entre arte y sociedad.

En síntesis, Bauman contribuyó a nuestra comprensión de que, a pesar de todos los cuestionamientos institucionales que realiza Duchamp, su acción sólo puede considerarse como un acto profundamente moderno. Es decir, que su labor se erige como un bastión del proyecto moderno: más exactamente, como una oportunidad de reinventar el mundo y privilegiar la subjetividad/libertad, por sobre la estructura social dominante. Por otra parte, la composición de los campos, por parte de Pierre 
Bourdieu, nos permitió ofrecer un panorama general de las estrategias de posicionamiento que los agentes sociales logran implementar para poseer la jerarquía de decisión de los parámetros de comprensión, permitiendo así comprender cómo Duchamp jugó con tales capacidades. Y Luhmann, por su parte, nos permitió comprender cómo los ready-made nos ofrecen la posibilidad de la creación de una observación de segundo orden, que traza nuevos e inesperados despliegues de auto-reflexión artística (evolución de un sistema artístico cada vez más complejo).

Como si fuera un tablero de ajedrez, la obra de Duchamp nos ofreció -y nos ofrece- múltiples posibilidades reflexivas. Y eso resulta, para toda disciplina, una gran oportunidad. Es de esperar que el arte contemporáneo pueda otorgarnos nuevos caminos de análisis como lo hiciera Duchamp en el siglo XX. El esfuerzo analítico aquí desplegado espera contribuir a esa misión y, por cierto, abrir en el país nuevos ámbitos analíticos y reflexivos.

\section{Bibliografía}

Adorno, Theodor W. y M. Horkheimer (2003). "Dialéctica de la ilustración", Editorial Trotta, Madrid.

Bauman, Zygmunt (2003). "Modernidad Líquida", Fondo de Cultura Económica, México.

(2005). "Legisladores e Intérpretes. Sobre la modernidad, la posmodernidad y los intelectuales", Editorial Universidad Nacional de Quilmes, Argentina.

(2007). “Arte, ¿Líquido?”, Editorial Sequitur, Madrid.

Becker, Howard (2008). "Los Mundos del Arte. Sociología del trabajo artístico", Editorial Universidad Nacional de Quilmes, Argentina.

Benjamin, Walter (2003). "La obra de arte en la época de su reproductibilidad técnica”, Editorial Ítaca, México.

Bourdieu, Pierre (1995). “Las reglas del arte: Génesis y estructura del campo literario", Editorial Anagrama, Barcelona.

(1997). "Razones prácticas. Sobre la teoría de la acción", Editorial Anagrama, Barcelona. 
(2002). "Pero, ¿quién creó a los creadores?", en "Cuestiones de Sociología", Editorial Istmo, Barcelona.

(2003a). “Creencia artística y bienes simbólicos. Elementos para una sociología de la cultura", Editorial Aurelia-Rivera, Buenos Aires.

(2003b). "Elementos de una teoría sociológica de la percepción artística", en "Campo de poder, Campo Intelectual”, Editorial Quadrata, Buenos Aires.

García Canclini, Néstor (1979). “La producción simbólica: teoría y método en sociología del arte", Editorial Siglo Veintiuno, México.

(2010). "La sociedad sin relato. Antropología y estética de la inminencia", Editorial Katz, Buenos Aires.

Danto, Arthur (2006). “Después el fin del arte. El arte contemporáneo y el linde de la historia", Editorial Paidós, Buenos Aires.

De la Fuente, Eduardo (2000). "Sociology and Aesthetics", European Journal of Social Theory; May2000, Vol. 3 Issue 2, p235, 1p.

Duvignaud, Jean (1988). "Sociología del arte”, Eds. Península, Barcelona.

Facuse, Marisol (2010). "Sociología del arte y América Latina: Notas para un encuentro posible", Revista UNIVERSUM, N ${ }^{\circ}$ 25, Vol.1, Universidad de Talca.

Foster, Hal (2001). "El retorno de lo real. La vanguardia a finales del siglo", Editorial Akal, Madrid.

Francastel, Pierre (1975). "Sociología del arte”, Alianza Editorial, Madrid.

Furió, Vincenç (2000). “Sociología del arte”, Editorial Cátedra, Madrid.

Gombrich, E.H (2007). "La historia del arte”, Editorial Phaidon, España.

Harrington, Austin (2004). "Art and Social Theory", Polity Press, Cambridge, UK.

Hauser, Arnold (1983). "Sociología del arte", Editorial Guadarrama, Barcelona.

Heinich, Nathalie (2001). "Lo que el arte aporta a la sociología”, Ediciones Sello Bermejo, México. (2002). "La sociología del arte", Ediciones Nueva Visión, Buenos Aires.

Huyssen, Andreas (2002). “Después de la gran división. Modernismo, cultura de masas, posmodernismo", Adriana Hidalgo Editora, Buenos Aires. 
Luhmann, Niklas (1982). "The differentiation of society", Columbia University Press, New York.

(1999). "El arte como mundo", en "Teoría de los sistemas sociales II (artículos)", Universidad de los Lagos, Chile.

(2005). "El arte de la sociedad", Editorial Herder-Universidad Iberoamericana, México D.F.

(2007). "Sociedad de la sociedad", Editorial Herder-Universidad Iberoamericana, México D.F.

Lyotard, Jean-Francois (1977). "Les transformateurs Duchamp", Editions Galilée, París, France.

dra, Madrid.

(1998). “La condición post-moderna”, Editorial Cáte-

Panofsky, Aaron (2003). "From Epistemology to the Avant-garde. Marcel Duchamp and the Sociology of Knowledge in Resonance", Theory, Culture \& Society, SAGE, Vol. 20(1): 61-92, London.

Pollock, Griselda (2007). "Thinking sociologically, thinking aesthetically. Between convergence and difference with some historical reflections on sociology and art history", History of the Human Sciences 2007; 20.

Ramírez, Juan Antonio (2000). "Duchamp. El amor y la muerte, incluso", Ediciones Siruela, Madrid, España.

Rancière, Jacques (2009). "El reparto de lo sensible. Estética y política", Ediciones Lom, Santiago de Chile.

Rojas, Sergio (2003). "La obra de arte en el proceso de autocomprensión de la sociedad moderna. Consideraciones filosóficas desde la sociología del arte", en "Imaginar la Materia. Ensayos de filosofía y estética", Editorial Arcis, Santiago.

Shiner, Larry (2004). "La invención del arte. Una historia cultural”, Editorial Paidós, Barcelona.

Wolff, Janet (1997). “La producción social del arte”, Eds. Istmo, Madrid.

Zolverg, Vera (2002). "Sociología de las artes", SGAE y Fundación Autor, Madrid. 